Yong Cheng

Chunyan Ji

Gangjun Zhai

Tianhui Fan*

http://dx.doi.org/10.21278/brod68402

ISSN 0007-215X

eISSN 1845-5859

\title{
INVESTIGATION OF HYDROELASTIC BEHAVIOR OF A PONTOON- TYPE VLFS DURING UNSTEADY EXTERNAL LOADS IN WAVE CONDITION USING A HYBRID FINITE ELEMENT-BOUNDARY ELEMENT (FE-ME) METHOD
}

\author{
UDC 629.5(05) Provided by the Author or by the Editor \\ Categorization suggested by the reviewers provided by the Editor
}

\begin{abstract}
Summary
The hydroelastic behavior of a pontoon-type VLFS subjected to unsteady external loads in wave condition is investigated in the context of the time-domain modal expansion theory, in which the boundary element method (BEM) based on time domain Kelvin sources is used for hydrodynamic forces and the finite element method (FEM) is adopted for solving the deflections of the VLFS. In this analysis, the interpolation-tabulation scheme is applied to assess rapidly and accurately the free-surface Green function in finite water depth, and the boundary integral equation of a quarter VLFS model is further established taking advantage of symmetry of flow field and structure. The VLFS is modeled as an equivalent solid plate based on the Mindlin plate theory. The coupled plate-water model is performed to determine the wave-induced responses and transient behavior under external loads such as a huge mass impact onto the structure and moving loads of an airplane, respectively. These results are verified with existing numerical results and experimental test. Then, the developed numerical tools are used in the study of the combined action taking into account of the mass drop/airplane landing as well as forward or reverse incident wave action. The deflections of the runway, the time history of vertical positions and the trajectory of the airplane are also presented through a systematic time-domain simulation, which illustrates the usefulness of the presently developed numerical solutions.
\end{abstract}

Key words: $\quad$ very large floating structure (VLFS); direct time-domain modal expansion method; hydroelastic response; Green function; interpolation-tabulation method; 
A direct time-domain simulation of hydroelastic behavior of a pontoon-VLFS during unsteady external loads in wave condition

\section{Introduction}

Mat-like very large floating structures (VLFS) are very flexible offshore structures widely regarded as an alternative option of utilizing ocean space for different applications such as floating airports, oil storage vessels, bridges, floating artificial island, etc. When a VLFS moves due to wave or moving load actions, the fluid surrounding the structure changes at the same time. On other hand, the pressure distribution over the body surface also changes in order to satisfy the Bernoulli's equation. If the pressure changes, the motions including rigid body motions and elastic deformations are affected. Thus, the fluid-structure interaction problem is called hydroelasticity and it is necessarily considered in order to obtain the vertical responses of a VLFS.

Numerous contributions have been performed on the research activities related to hydroelastic analysis of VLFS. Watanabe et al. [1] and Eatock Taylor [2] presented a review of these methods on the simulation of hydroelastic responses of VLFS. One way to tackle this problem is to use an analytical approach given by Wu et al. [3], Watanabe et al. [4], Pham et al. [5], and Karmakar and Soares [6]. They divided the whole fluid domain into multiple regions and applied the eigenfunction expansion-matching method for obtaining the waveinduced deflections of the VLFS. Based on Mindlin thick plates theory and Wiener-Hopf technique, Zhao et al. [7] presented the dynamical behavior of floating elastic plates acted upon by a localized external load (periodic load). If these analytical methods are used, the computational time and memory capacity for a VLFS are non-issue. However, the solved drawback is only applied to simple geometries such as a rectangular plate and a circular plate. Another way to obtain the hydroelastic responses of VLFS is by using a numerical method. The numerical solutions can be applied to more any shape of VLFS, and the boundary element method (BEM) [8-11], the finite-element method (FEM) [12-18], and the hybrid finite element-boundary element (FE-BE) method [19-22] have been presented in previous studies.

Among various numerical studies, the frequency domain wave-induced behavior has been commonly investigated when determining the hydroelastic response amplitude operator (RAO) of the floating body and pertinent response parameters in a steady state condition. However, in the real situation, we would often like use some time series of the responses, and non-harmonic external loads such as imposed by a huge mass impact onto the structure, landing and taking off of an airplane, can induce the transient behavior of the VLFS and may affect the serviceability of the VLFS. Watanabe et al. [12], Qiu and Liu [13], and Shin et al. [14] applied the finite element methods (FEM) to the transient response analysis of a VLFS due to impulsive landing/takeoff of an airplane, however, the response analysis is required for a few seconds and this simulation model is very much simplified in the treatment of structure or fluid. Kashiwagi $[8,9]$ developed an indirect time-domain method for calculating transient responses of a VLFS, in which the hydrodynamic effect is evaluated from good performance in the computation of the memory-effect function. Lee and Choi [22] proposed a boundary element-finite element (BE-FE) hybrid method to solve the transient responses indirectly by using transient equations, which are derived from the Fourier inverse transform of harmonic equations of motion and the causality condition. Based on the BE-FE combined method, Endo [23 and 24] simulated the transient behavior of a VLFS subjected simultaneously to takeoff/landing and regular wave load. These mentioned studies provide enlightening contributions in the research activities related to external loads on the VLFS but some difficulties in carrying out their time-domain simulation give restriction on the mathematical model, i.e. the assumption of both small structural motion and wave amplitude, the integration of memory-effect function, and the accurate evaluation of hydrodynamic coefficients. Our approach, instead, is to obtain directly the wave-induced responses and transient phenomena 
behavior of a pontoon-VLFS during unsteady external

loads in wave condition

by using a direct time-domain modal expansion method. In this analysis, the numerical model and scheme developed by the same authors [21 and 25] for a VLFS edged with dual antimotion plates under wave action (without any unsteady external loads) is extended to incorporate the non-harmonic external loads. The method takes account of applying the timedomain free-surface Green functions in the hydrodynamic diffraction and radiation problems whereas the element used to establish the plate model is the non-conforming quadraticserendipity (NC-QS) Mindlin plate element [20]. Such a Mindlin plate element does not suffer from spurious modes and shear locking phenomenon, and considers the effects of shear deformation and rotary inertia, which are neglected in the Classical thin plate theory. The fluid-structure interaction is investigated using a hybrid finite element-boundary element (FEME) method, where the FEM is used to solve the coupled plate-water equation whereas the BEM is used to handle the water integral boundary equation. Compared with FEM, the modal expansion method can clearly reduce the number of unknown amplitudes which can be obtained directly from a solution of simultaneous equations at each time step.

Fast and accurate calculation is necessary for overcoming this difficulty of large CPU time and memory size of computer. Utsunomiya et al. [26] has developed the multipole expansion methods for hydroelastic analysis of a VLFS. Kagemoto et al. [27] presented a substructural method that accelerates computation without an appreciable loss of accuracy. Dai [28] has expanded the precorrected-FFT method to hydroelastic analysis. However, their calculation models only satisfy the frequency domain studies for the wave-induced hydroelastic response. Huang [29] has put forth a feasible technique to tackle the time-free surface Green functions in infinite water depth, however, the VLFS commonly is placed in finite water depth. Thus the authors derive the expression of the time-domain free-surface Green functions and its spatial derivatives in finite water depth, which with sufficient accuracy are rapidly evaluated by using an interpolation-tabulation method. The low number of elements also is an important technique to reduce the memory and CPU time when the pressure distribution is obtained by using BEM. According to the symmetry of the VLFS structure and the fluid field [30,31], this paper is concerned with numerical simulations of boundary integral equations of a quarter VLFS model.

\section{Mathematical formulation}

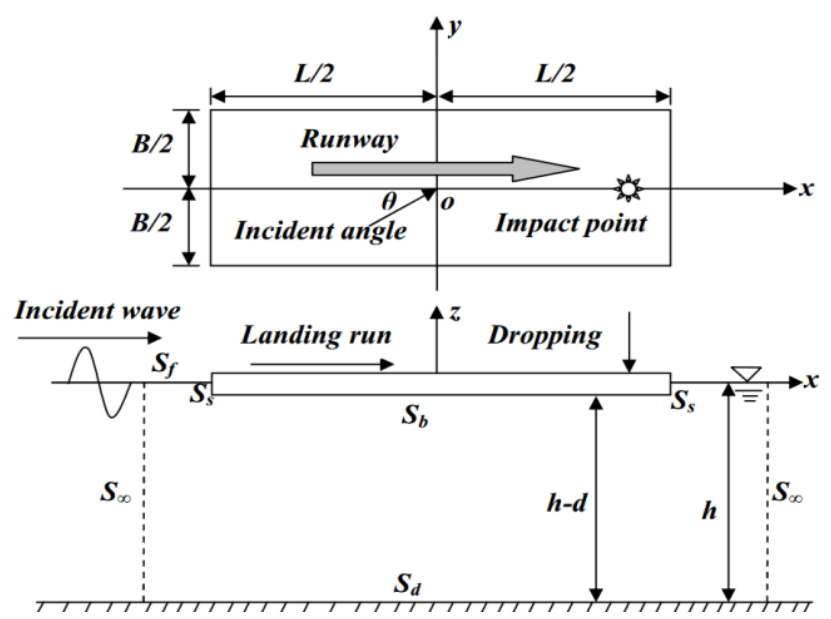

Fig.1 The fluid-structure system and coordinate system

For the time domain elastic responses of a pontoon-type VLFS in finite water depth, Fig. 1 shows the fluid-structure problem and Cartesian coordinate system. In the model, the zaxis is pointing upwards, and the $x-y$ plane is on the mean position of the free surface, where $h$ is the water depth, $A$ is the amplitude of the incident wave. The whole fluid domain is defined at $\Omega$ which contains the bottom of the VLFS $S_{b}$, side of the VLFS $S_{s}$, the free surface 
$S_{f}$, the seabed $S_{d}$ and the infinite cylindrical surface $S_{\infty}$. The VLFS has a length $L$, width $B$, height $h v$, and $d$ is the draft of the VLFS in $\mathrm{z}$ direction. The problem at hand is to determine the modal deflections under external loads combined action of incident wave.

Fluid is assumed to be ideal, so that a velocity potential exists and is governed by the Laplace equation:

$$
\nabla^{2} \Phi(x, y, z, t)=0
$$

and the boundary conditions are satisfied as the following:

$$
\begin{array}{ll}
\frac{\partial^{2} \Phi(x, y, z, t)}{\partial t^{2}}+g \frac{\partial \Phi(x, y, z, t)}{\partial z}=0 & \text { on } S_{f}, t>0 \\
\frac{\partial \Phi_{I}(x, y, z, t)}{\partial n}+\frac{\partial \Phi_{s}(x, y, z, t)}{\partial n}=V_{n} & \text { on } S_{b}+S_{s}, t>0 \\
\frac{\partial \Phi_{s}(x, y, z, t)}{\partial n}=0 & \text { on } S_{d}, t>0 \\
\Phi_{s}(x, y, z, t), \Phi_{s t}(x, y, z, t), \nabla \Phi_{s}(x, y, z, t) & \rightarrow 0 \quad \text { on } S_{\infty}, t>0 \\
\Phi_{s}(x, y, z, t)=\Phi_{s t}(x, y, z, t)=0 & \text { on } S_{f}, t=0
\end{array}
$$

where $\Phi_{I}(x, y, z, t)$ and $\Phi_{S}(x, y, z, t)$ are the incident and scattering potential, respectively. $g$ is the gravitational acceleration, $V_{n}$ is the normal velocity of the structure, $n$ is a unit normal vector (the positive direction points out of the fluid domain), $\Phi_{s t}(x, y, z, t)$ represent the time derivative of scattering potential.

By assuming the plate material to be isotropic and obey the Hooke's law, the motion of the floating body is governed by the equation of Mindlin thick plate [32], i.e.

$$
D\left(B_{1}+B_{2}\right) \overrightarrow{\mathbf{W}}+\rho_{s} \omega^{2} B_{3} \overrightarrow{\mathbf{W}}=-\overrightarrow{\mathbf{F}}
$$

where $\overrightarrow{\mathbf{W}}$ is the displacement vector including of the vertical deflection $W(x, y, t)$, the rotation $\Psi_{x}(x, y, t)$ about the $y$-axis and the rotation $\Psi_{y}(x, y, t)$ about the $x$-axis. $D$ and $\rho_{\text {s }}$ denote the bending rigidity and the structural density, respectively. The differential operators $B_{1}$ and $B_{2}$ and the constant matrix $B_{3}$ are defined in Ref. [18]. The loads vector $\overrightarrow{\mathbf{F}}$ comprises the hydrostatic pressure $-\rho g W$, hydrodynamic pressure $-\rho \frac{\partial \Phi(x, y,-d, t)}{\partial t}$ at the bottom of the VLFS, and the arbitrary external time-dependent loads $P_{E}$ such as a weight drop load, an airplane landing or take off acting on the deck of the VLFS, i.e.

$$
\overrightarrow{\mathbf{F}}=\left\{\begin{array}{c}
-\rho g W(x, y, t)-\rho \frac{\partial \Phi(x, y, t)}{\partial t}-P_{E} \\
0 \\
0
\end{array}\right\}
$$

In the present case, the VLFS is not constrained in the vertical elastic displacement along its edges, the following boundary conditions for a free edge must be satisfied:

Bending/Twisting moment $\left[D_{f}\right]\{\chi\}=0 \quad x= \pm L / 2, y= \pm B / 2$

Shear force $\left[D_{s}\right]\{\gamma\}=0 \quad x= \pm L / 2, y= \pm B / 2$

where $\left[D_{f}\right],\left[D_{s}\right],\{\chi\}$ and $\{\gamma\}$ are the flexural elasticity matrix, the shear elasticity matrix, the flexural strain and the shear strain, respectively, and have been given in Ref. [20]. 
A direct time-domain simulation of hydroelastic behavior of a pontoon-VLFS during unsteady external loads in wave condition

\section{Method of solutions}

The solution for the hydroelastic responses of the VLFS involves solving the platewater motion equation and the water boundary integral equation by applying the hybrid finite element- boundary element (FE-ME) method. Here, the FEM with non-conforming quadraticserendipity (NC-QS) Mindlin plate element is applied to solving the time series of the plate responses whereas the BEM is used to obtaining the water scattering potential.

\subsection{Fluid part}

The boundary value problems given by Eqs. (1)-(6) can be solved by using the Green's function method. If the free-surface Green's function satisfying the boundary conditions given by Eqs (2), (4), (5), and (6) is considered, the boundary integral equation for the scattering potential can be derived as follows:

$$
\begin{aligned}
& \alpha \Phi_{S}(Q, t)+\iint_{S_{b(t)}+S_{s(t)}} \Phi_{S}(P, t) \frac{\partial G^{0}(P, Q)}{\partial n_{p}} d S_{p}=\iint_{S_{b(t)}+S_{s(t)}} G^{0}(P, Q) \frac{\partial \Phi_{S}(P, t)}{\partial n_{p}} d S_{p} \\
& +\int_{0}^{t} d \tau \iint_{S_{b(\tau)}+S_{s(\tau)}}\left[\Phi_{S}(P, \tau) \frac{\partial G_{\tau}^{f}(P(\tau), Q(t), t-\tau)}{\partial n_{p}}-G_{\tau}^{f}(P(\tau), Q(t), t-\tau) \frac{\partial \Phi_{S}(P, \tau)}{\partial n_{p}}\right] d S_{p} \\
& +\frac{1}{g} \int_{0}^{t} d \tau \iint_{C b(\tau)}\left[\Phi_{S}(P, \tau) G_{\tau \tau}^{f}(P(\tau), Q(t), t-\tau)-G_{\tau}^{f}(P(\tau), Q(t), t-\tau) \frac{\partial \Phi_{S}(P, \tau)}{\partial \tau}\right] \cdot V_{n}(P, \tau) d S_{p}
\end{aligned}
$$

where $\alpha$ represents the solid angle, $Q\left(x_{0}, y_{0}, z_{0}\right)$ and $P(x, y, z)$ represent the source and field point, respectively, and $C b(t)$ represents the instantaneous waterline of the intersection between the body and the free-surface. For Green function $G(P, t, Q, \tau)$, it can be expressed by the superposition of instantaneous term $G^{0}$ and memory term $G^{f}$ [34]:

$$
G(P, t, Q, \tau)=G^{0}(P, Q)+G^{f}(P, t, Q, \tau)
$$

where the instantaneous term $G^{0}$ and memory term $G^{f}$ are given in the form, respectively

$$
G^{0}=\frac{1}{r}+\frac{1}{r_{2}}-2 \int_{0}^{\infty} e^{-k h} \frac{\cosh k\left(z_{0}+h\right)}{\cosh k h} \cosh k(z+h) J_{0}(k R) d k
$$

$$
G^{f}=2 \int_{0}^{\infty} \frac{\cosh k(z+h)}{\cosh k h \sinh k h} \cosh k\left(z_{0}+h\right)\{1-\cos [(t-\tau) \sqrt{g k \tanh k h}]\} J_{0}(k R) d k
$$

$J_{0}$ is the Bessel function of the first kind, order zero, $R$ denotes the horizontal distance between field and source point, $r$ denotes the distance between field and source point, and $r_{2}$ denotes the distance between field and the mirror image of the source field about seabed. We non-dimensionalise the spatial parameters using $X=R / h, Y=-z_{0} / h, Z=-z / h$ and the time parameter using $T=(t-\tau)(g / h)^{1 / 2}$. The instantaneous term $G^{0}$ is evaluated using the method of Cheng et al. [25], and the first order time derivative $G_{\tau}^{f}$ is expressed in the form:

$$
G_{\tau}^{f}=-g^{1 / 2} h^{-3 / 2}[F(X, Y-Z, T)+F(X, 2-Y-Z, T)]
$$

where

$$
\begin{aligned}
F(X, V, T) & =\int_{0}^{\infty} \frac{\sqrt{k \tanh k}}{\cosh k \sinh k} \sin (T \sqrt{k \tanh k}) \cosh (k V) J_{0}(k X) d k \\
& =2 \int_{0}^{\infty} \sqrt{k \tanh k} \sin (T \sqrt{k \tanh k}) \frac{e^{k(V-2)}+e^{-k(V+2)}}{1-e^{-4 k}} J_{0}(k X) d k
\end{aligned}
$$


loads in wave condition

The rate of convergence of the integral in Eq. (21) can be accelerated by adding and subtracting the appropriate function $F_{\infty}$ which may be given in the form

$$
\begin{aligned}
F_{\infty}(X, V, T) & =\lim _{k \rightarrow \infty} F(X, V, T) \\
& =2 \int_{0}^{\infty} \sqrt{k} \sin (T \sqrt{k}) e^{k(V-2)} J_{0}(k X) d k
\end{aligned}
$$

where the vertical coordinate $V$ is restricted to the fluid domain $(-1,2)$.

If the spherical coordinate is adopt, the non-dimensional parameters on $r$ are defined by

$$
r=\left[X^{2}+(V-2)^{2}\right]^{1 / 2}, \tau=T / r^{1 / 2}, k=k r, V-2=r \cos \theta, X=r \sin \theta
$$

where $\tau$ and $\theta$ are lies in the interval $(0, \infty)$ and $(0,2 \pi)$. Thus, we will reduce three arguments to two arguments by substituting Eq. (18) into Eq. (17). The function $F_{\infty}$ and its spatial derivatives are obtained:

$$
\begin{aligned}
& F_{\infty}=2 r^{-\frac{3}{2}} \operatorname{Im}\left\{\int_{0}^{\infty} \sqrt{k} e^{-k \cos \theta} e^{i \tau \sqrt{k}} J_{0}(k \sin \theta) d k\right\} \\
& F_{\infty X}=-2 r^{-\frac{5}{2}} \operatorname{Im}\left\{\int_{0}^{\infty} k^{3 / 2} e^{-k \cos \theta} e^{i \tau \sqrt{k}} J_{1}(k \sin \theta) d k\right\} \\
& F_{\infty V}=2 r^{-\frac{5}{2}} \operatorname{Im}\left\{\int_{0}^{\infty} k^{3 / 2} e^{-k \cos \theta} e^{i \tau \sqrt{k}} J_{0}(k \sin \theta) d k\right\} \\
& F_{\infty T}=2 r^{-2} \operatorname{Re}\left\{\int_{0}^{\infty} k e^{-k \cos \theta} e^{i \tau \sqrt{k}} J_{0}(k \sin \theta) d k\right\}
\end{aligned}
$$

Since the integrands in Eqs. (19)-(22) exhibit slowly convergence and highly oscillation, the $F_{\infty}$ and its spatial derivatives can be approximated using series expansion, asymptotic expansion or Filon quadrature in terms of the values of parameter $\tau$ [29 and 34]. The function $F-F_{\infty}$ may be solved directly in a straightforward manner due to oscillatory elimination, thus $F(X, V, T)$ is obtained:

$$
F(X, V, T)=F_{\infty}+F-F_{\infty}
$$

Then the boundary surface of Eq. (11) is discretized into a number of elements using a standard procedure known as the BEM. Within the boundary elements, physical variables are interpolated by the shape functions [35], which represent the geometry of each element. In the integration process, the scheme using trapezoidal approximation is applied to the convolution integral. Once Eq. (11) is solved, the time history of fluid dynamic pressure in Eq. (8) can be obtained at any position.

\subsection{Structure part}

In order to use the FEM for solving the plate equation, Eq. (7) is transformed into an equivalent motion equation by using first-order shear deformation plate theory based on Hamilton's principle. The shear correction shear factor is taken as 5/6 according to Ref. [20, 36-38]. The VLFS model is approximated by a number of the NC-QS Mindlin thick plate elements, in which additional non-conforming basis functions are added to the bending rotations, and thus each element does not exhibit spurious modes and shear locking phenomena. By transforming the coupled plate-water Eq. (7) into the equivalent variational equation and minimizing energy functional, we obtain the global form of coupled plate-water equation 


$$
[M]\{\ddot{\mathbf{W}}\}+[C]\{\dot{\mathbf{W}}\}+\left(\left[K_{f}\right]+\left[K_{s}\right]+\left[K_{r e}\right]\right)\{\mathbf{W}\}=\left\{F_{w}\right\}+\left\{F_{E}\right\}
$$

where $[C]$ is the global viscous damping matrix, which can be neglected in context of potential theory; $[M],\left[K_{f}\right],\left[K_{s}\right],\left[K_{r e}\right],\left\{F_{w}\right\}$ and $\left\{F_{E}\right\}$ are the global mass matrix, the global flexural stiffness matrix, the global shear stiffness matrix, the global restoring force matrix, the global wave force vector and the global external load force vector, respectively. All of these entities can be assembled from corresponding single element matrix $[m]_{e},\left[k_{f}\right]_{e},\left[k_{s}\right]_{e}$, $\left[k_{r e}\right]_{e},\left\{f_{w}\right\}_{e}$ and $\left\{f_{E}\right\}_{e}$, i.e.

$$
\begin{aligned}
& {[M]=\sum_{e}[m]_{e}=\sum_{e} \iint_{e} \rho_{s}[N]^{T}\left[\begin{array}{ccc}
h_{s} & 0 & 0 \\
0 & h_{s}^{3} / 12 & 0 \\
0 & 0 & h_{s}^{3} / 12
\end{array}\right][N] d s_{e},} \\
& {\left[K_{f}\right]=\sum_{e}\left[k_{f}\right]_{e}=\sum_{e} \iint_{e}\left[B_{f}\right]^{T}\left[D_{f}\right]\left[B_{f}\right] d s_{e},} \\
& {\left[K_{s}\right]=\sum_{e}\left[k_{s}\right]_{e}=\sum_{e} \iint_{e}\left[B_{s}\right]^{T}\left[D_{s}\right]\left[B_{s}\right] d s_{e},} \\
& {\left[K_{r e}\right]=\sum_{e}\left[k_{r e}\right]_{e}=\sum_{e} \rho g \iint_{e}[N]^{T}\left[\begin{array}{ccc}
1 & 0 & 0 \\
0 & 0 & 0 \\
0 & 0 & 0
\end{array}\right][N] d s_{e},} \\
& \left\{F_{w}\right\}=\sum_{e}\left\{f_{w}\right\}_{e}=\sum_{e} \iint_{e}[N]^{T}[N]\left\{-\rho \frac{\partial\left(\Phi_{I}+\Phi_{S}\right)}{\partial t}\right\} d s_{e}, \\
& \left\{F_{E}\right\}=\sum_{e}\left\{f_{E}\right\}_{e}=\sum_{e} \iint_{e}[N]^{T}[N]\left\{P_{E}\right\} d s_{e} .
\end{aligned}
$$

where $[N]$ is the NC-QS element interpolation function matrix; superscript $T$ denotes matrix inversion; $e$ means numerical formulation on elements; $\left[B_{f}\right]$ is the flexural strain-displacement matrix whereas $\left[B_{s}\right]$ is the shear strain-displacement matrix as found in [18]. Here, the global restoring force matrix $K_{r e}$ is only related to the hydrostatic pressure term in Eq. (8), and ignores the effect of rigid body motion [39 and 40]. The displacement vector $\{\mathbf{W}\}$ is expressed in terms of modal expansion as follows:

$$
\{\mathbf{W}\}=[f]\{\zeta(t)\}=\left[\begin{array}{l}
f_{i j}^{w} \\
f_{i j}^{\psi_{x}} \\
f_{i j}^{\psi_{y}}
\end{array}\right]\left\{\zeta_{j}\right\}
$$

Here $\zeta_{j}$ is the $j$ th generalized modal coordinate; subscript $i$ denotes the node number and $j$ denotes the mode number; $f_{i j}{ }^{w}, f_{i j}^{\psi_{x}}$ and $f_{i j}^{\psi_{y}}$ are $j$ th natural modes corresponding to vertical displacement, rotation about the $y$-axis and rotation about the $x$-axis, respectively, and they can be obtained by solving the eigenvector equation

$$
\left(\left[K_{f}\right]+\left[K_{s}\right]\right)[f]=[\lambda][M][f]
$$

where $[\lambda]$ is the square diagonal matrix of $j$ th natural circular frequency.

By substituting Eq. (31) into VLFS-water Eq. (24) and premultiplying both sides of the Eq. (24) by $[f]^{T}$, we can obtain a conventional set of equations given by

$$
[G M]\{\ddot{\zeta}\}+[G C]\{\dot{\zeta}\}+[G K]\{\zeta\}=\left\{G F_{w}\right\}+\left\{G F_{E}\right\}
$$

with 
loads in wave condition

$$
\begin{aligned}
& {[G M]=[f]^{T}[M][f]} \\
& {[G C]=[f]^{T}[C][f]} \\
& {[G K]=[f]^{T}\left(\left[K_{f}\right]+\left[K_{s}\right]+\left[K_{r e}\right]\right)[f]} \\
& \left\{G F_{w}\right\}=[f]^{T}\left\{F_{w}\right\},\left\{G F_{E}\right\}=[f]^{T}\left\{F_{E}\right\}
\end{aligned}
$$

where $[G M],[G C],[G K],\left\{G F_{w}\right\}$ and $\left\{G F_{E}\right\}$ are the generalized mass matrix, the generalized damping matrix, generalized stiffness matrix, generalized wave force vector and generalized external load force vector, respectively.

The second order linear differential equations for modal amplitudes shown in Eq. (33) are solved at each time step using the fourth order Runge-Kutta method. Then the modal responses are summed up to obtain the total response.

\section{Fast algorithm}

\subsection{Interpolation-tabulation method}

Accurate and fact computation of the Green function and its derivations is important for saving the CPU time and memory of the computer. The interpolation-tabulation method is applied to the solutions of $F_{\infty}$ and $F$ - $F_{\infty}$ in Eq. (23) as follows.

Referring to the Eqs. (19)-(22), the three variables $X, V, T$ of the function $F_{\infty}$ are changed to two arguments $\tau$ and $\cos \theta$, which are divided 800 and 200 parts, respectively. The solutions of $F_{\infty}$ and its partial derivatives for $\cos \theta<0.7$ which are efficient in the context of section 3.2 are described by Huang [29], else the Filon integral scheme is determined to calculate directly. During solving the Eq. (11), the bilinear interpolation scheme was applied to the effective approximation of $F_{\infty}$ and its' derivation.

However, the $F-F_{\infty}$ function has three arguments $V, X$ and $T$. Here, the space nondimensional parameters $V$ and $X$ are restricted to the region $(-1,2)$ and $(0,20)$, the time nondimensional parameters $T$ lies in the interval $(0,20)$. First seven $X$ - $T$ planes are adopted in $V$ direction. Next, every $X-T$ plane is divided 40 parts in $X$ and $T$ direction, respectively. The slowly-varying function $F-F_{\infty}$ and its' derivations can be calculated by using Gaussian integration, then the tri-linear interpolation scheme is applied to the effective approximation in Eq. (11).

\subsection{Symmetry of structure}

With the discretization of the constant boundary elements, the Eq. (11) may be expressed as form of the linear equations

$$
[\boldsymbol{A}]\left\{\boldsymbol{\Phi}_{S}\right\}=\{\boldsymbol{B}\}
$$

Considering symmetry of the VLFS about x-z plane and y-z plane shown in Figure 2, the matrix $[\boldsymbol{A}]$, vector $\left\{\boldsymbol{\Phi}_{S}\right\}$ and $\{\boldsymbol{B}\}$, may be divided as follows:

$$
\left[\begin{array}{llll}
A_{11} & A_{12} & A_{13} & A_{14} \\
A_{21} & A_{22} & A_{23} & A_{24} \\
A_{31} & A_{32} & A_{33} & A_{34} \\
A_{41} & A_{42} & A_{43} & A_{44}
\end{array}\right]\left\{\begin{array}{l}
\Phi_{S}^{1} \\
\Phi_{S}^{2} \\
\Phi_{S}^{3} \\
\boldsymbol{\Phi}_{S}^{4}
\end{array}\right\}=\left\{\begin{array}{l}
\boldsymbol{B}_{1} \\
\boldsymbol{B}_{2} \\
\boldsymbol{B}_{3} \\
\boldsymbol{B}_{4}
\end{array}\right\}
$$

It is noted that the subscripts in matrix $[\boldsymbol{A}]$ and vector $\{\boldsymbol{B}\}$ or superscripts in vector $\left\{\boldsymbol{\Phi}_{\mathbf{s}}\right\}$ denote the related region of matrix or vector, for example, $\left[\boldsymbol{A}_{11}\right]$ is the sub-matrix 
corresponding to the region 1 , and $\left[\boldsymbol{A}_{12}\right]$ is the sub-matrix corresponding to the region 1 and 2 , and so on. In addition, the symmetric relationships for the matrix $[\boldsymbol{A}]$ may be formulated:

$$
\begin{aligned}
& A_{11}=A_{22}=A_{33}=A_{44} \\
& A_{12}=A_{21}=A_{34}=A_{43} \\
& A_{13}=A_{31}=A_{24}=A_{42} \\
& A_{14}=A_{41}=A_{23}=A_{32}
\end{aligned}
$$

In order to reduce the dimensions of the matrix, the conversions is obtained by taking

$$
\left\{\widehat{\boldsymbol{\Phi}}_{S}\right\}=[\boldsymbol{E}]\left\{\boldsymbol{\Phi}_{S}\right\},\{\widehat{\boldsymbol{B}}\}=[\boldsymbol{E}]\{\boldsymbol{B}\}, \quad[\boldsymbol{I}]=\frac{1}{\beta}[\boldsymbol{E}]^{2}
$$

where the matrix $[\boldsymbol{E}]$ is a transition matrix and the constant coefficient $\beta=4$. After substituting Eq. (44) into Eq. (38), the linear system of equations may be obtained for each region from 1 to 4 defined in Fig. 2 and they have been given by Ref. [11].

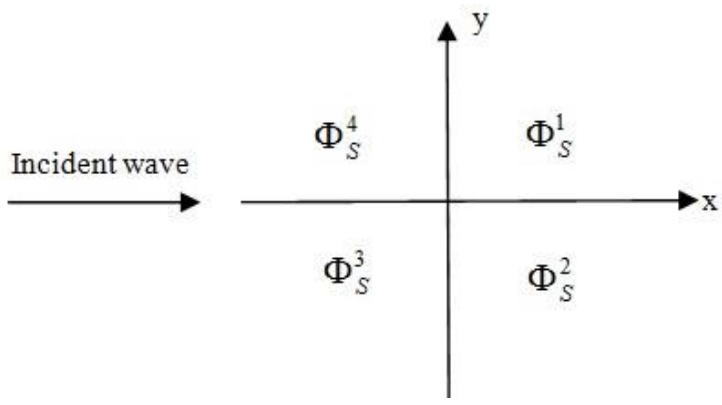

Fig.2 Sketch of the district of symmetry

\section{Results and discussions}

\subsection{Accuracy in the interpolation-tabulation method}

Before starting numerical simulations, it is necessary to confirm good performance in the computation of the time domain free-surface Green functions and its spatial derivatives in finite water depth.

In order to examine the validity of the interpolation-tabulation method, computations of the non-dimensional functions $F_{\infty}, F-F_{\infty}$ and their time derivatives are performed for $T=10$, $V=2$ and compared with corresponding results obtained from Newman. The values are shown in Figs. 3 and 4. Obviously, the interpolation-tabulation method can give reliable evaluations which are smooth and good agreement with the Newman's values.
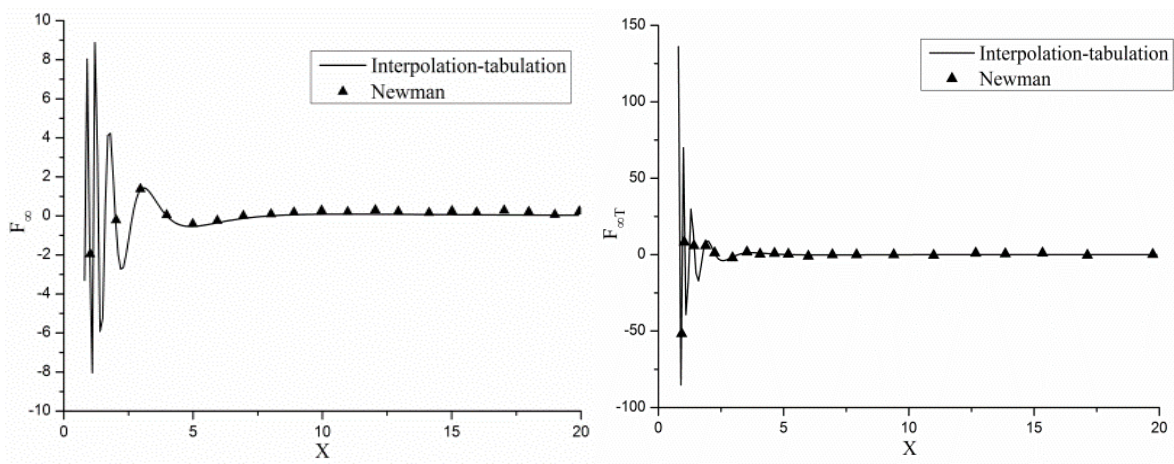

Fig. 3 Comparison of $F_{\infty}$ and $F_{\infty T}$ by the interpolation-tabulation method with Newman 
A direct time-domain simulation of hydroelastic behavior of a pontoon-VLFS during unsteady external loads in wave condition
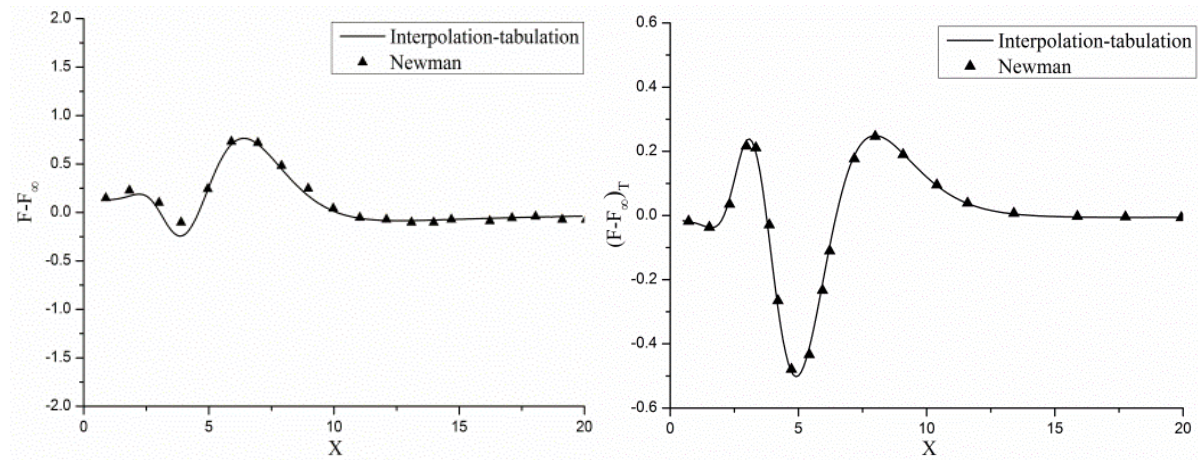

Fig.4 Comparison of $\mathrm{F}-\mathrm{F}_{\infty}$ and $\left(\mathrm{F}-\mathrm{F}_{\infty}\right)_{\mathrm{T}}$ by the interpolation-tabulation method with Newman

\subsection{Hydroelastic response of VLFS in wave conditions}

In order to validate the present formulation and method in the foregoing sections, we first performed the hydroelastic response of VLFS for the loads of regular wave conditions by using the direct time-domain method and the numerical solutions are compared with the analytical results solved by $\mathrm{Wu}$ et al. [3] and experimental tests obtained by Utsunomiya et al. [19]. The pertinent information for the VLFS design used by Utsunomiya et al. [19] is listed in Table 1. Based on convergence tests, it is found that the ratio of element size to wavelength must be smaller than 0.1 , and the vertical deflection obtained is confirmed to be stable and periodic after three cycles of the wave period. In addition, The time step defined as $T / 60$ and modes $N=30$ are sufficient for the deflections to converge of VLFS.

Figs. 5 and 6 show the distribution of vertical displacement amplitude and bending moment amplitude to incident regular wave with wave periods of $T=1.429 \mathrm{~s}$ and $2.875 \mathrm{~s}$. In this analysis, the element size to wavelength ratio must be smaller than 0.1 after checking the convergence, and the vertical deflection obtained is confirm to be stable and periodic after three cycles of the wave period. In the two cases, the good agreement is shown between our numerical solutions and $\mathrm{Wu}$ et al.'s analytical solutions, and the correlation between the present method and the experimental data is reasonable. The slight discrepancy between the calculated and measured data can be attributed to the possibly un-captured physics (such as vortices and wave breaking), the fluid viscosity, the energy loss induced by the gap between structure and tank wall, inherent nonlinear effects of wave, and the instrument accuracy.

Table 1 Principal details of the VLFS model and sea states

\begin{tabular}{|l|l|}
\hline Input data & Value \\
\hline Total length of the VLFS $(\mathrm{m})$ & 10 \\
\hline Total width of the VLFS $(\mathrm{m})$ & 0.5 \\
\hline Thickness of the VLFS $(\mathrm{m})$ & 0.038 \\
\hline Draft $(\mathrm{m})$ & 0.00836 \\
\hline Young's modulus (MPa) & 103 \\
\hline Poisson's ratio & 0.3 \\
\hline Water depth (m) & 1.1 \\
\hline Wave incident angle & $0^{0}$ \\
\hline Wave period (s) & $1.429,2.875$ \\
\hline Wave height $(\mathrm{m})$ & 0.02 \\
\hline
\end{tabular}


A direct time-domain simulation of hydroelastic behavior of a pontoon-VLFS during unsteady external loads in wave condition
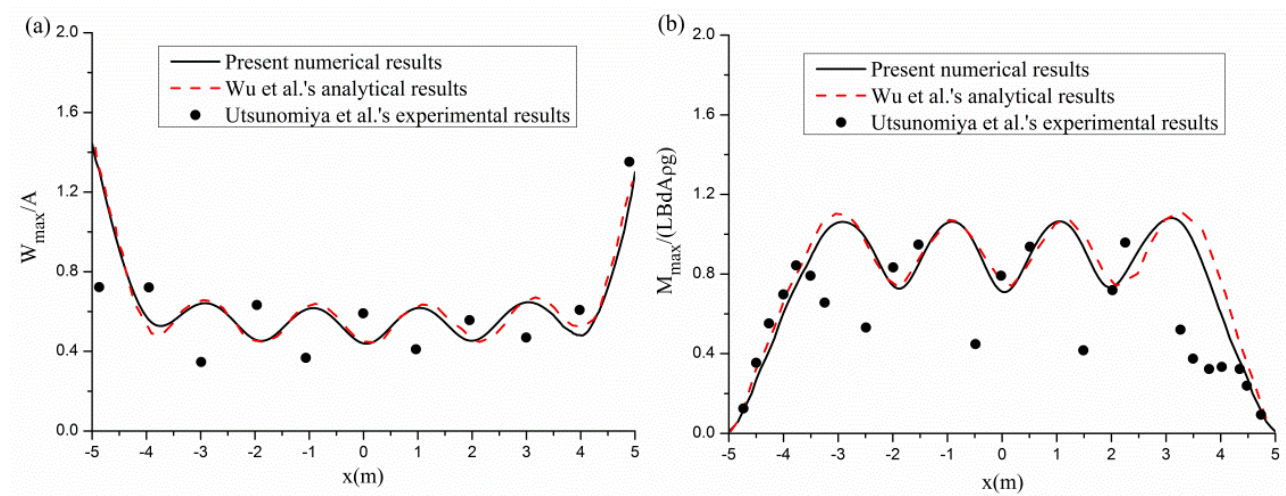

Fig.5 Comparison of numerical and experimental results for Utsunomiya et al.'s VLFS for regular wave period $\mathrm{T}=1.429$ (a) vertical displacement amplitude (b) bending moment amplitude
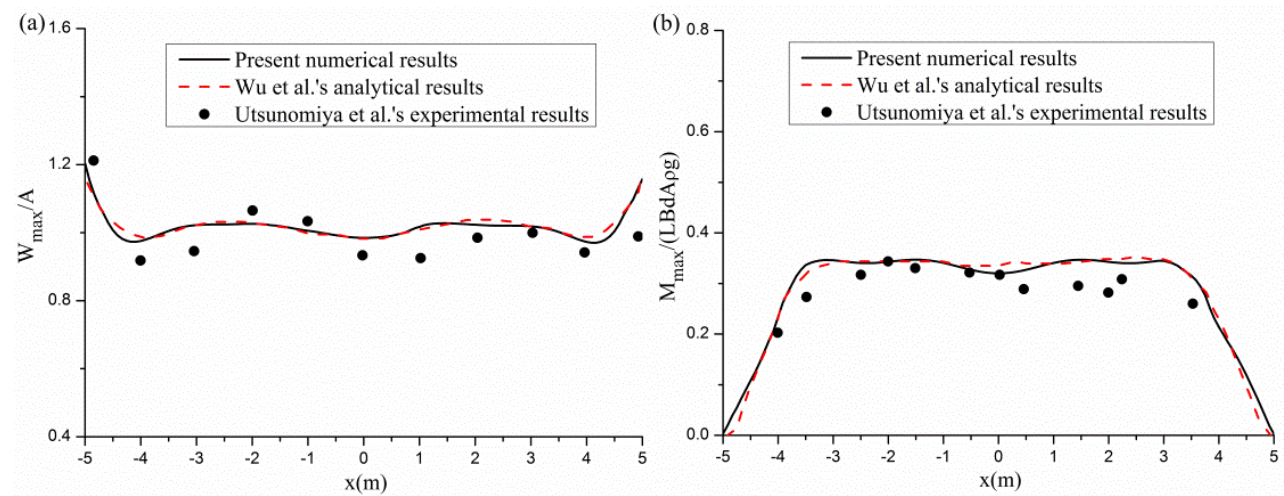

Fig.6 Comparison of numerical and experimental results for Utsunomiya et al.'s VLFS for regular wave period $\mathrm{T}=2.875$ (a) vertical displacement amplitude (b) bending moment amplitude

\subsection{Drop test in the still water}

The transient phenomena of a weight drop test are then implemented on the VL-10 model, corresponding to the experiment conducted by Endo and Yago [24]. In this experiment, the model has a length $9.75 \mathrm{~m}$, width $1.95 \mathrm{~m}$, thickness $0.0163 \mathrm{~m}$ and bending rigidity $8985.62 \mathrm{Nm}$. The weight of $W m=196 \mathrm{~N}$ was dropped from a height of $0.12 \mathrm{~m}$ onto the "hit point" (see Fig. 7) and then the impact load $F_{0}(t)$ can be obtained:

$$
F_{0}(t)=W m \cdot a
$$

where $a$ denotes the acceleration of the weight during the impact and it has been measured from Kashiwagi [8]. The external pressure distribution $P_{E}$, appearing in Eq. (30) can be expressed as

$$
P_{E}(x, y, t)=F_{0}(t) \cdot \delta\left(x-x_{p}\right) \cdot \delta\left(y-y_{p}\right)
$$

where $\left(x_{p}, y_{p}\right)$ is the coordinate of the hit point.

Fig. 8 comparatively shows the time histories of the vertical displacement at measured points Z1-Z9 indicated in Fig. 7 among the present method, the numerical results solved by Kashiwagi [8] and experimental tests obtained by Endo and Yago [24]. The present numerical results correlate reasonably with the Kashiwagi's numerical solutions and Endo and Yago's experimental results. It is also interesting that the deflections by the present method near the impact point, such as Z1 and Z2, are closer to the measurements than the Kashiwagi's numerical results. This may be due to the difference in fluid pressure computation between the direct domain method by considering free-surface Green function and the indirect domain method by using the convolution integral of frequency impulse function. 


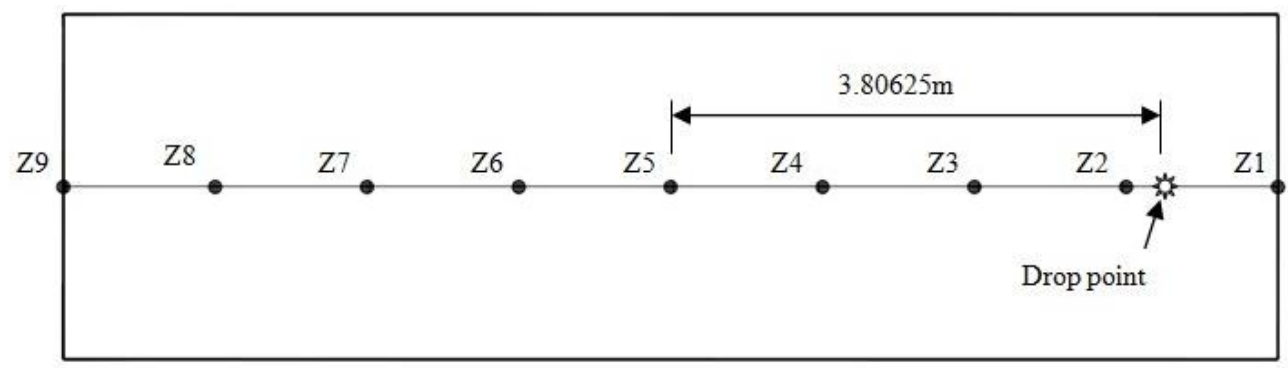

Fig. 7 The position of the measured points and hit point in the drop test
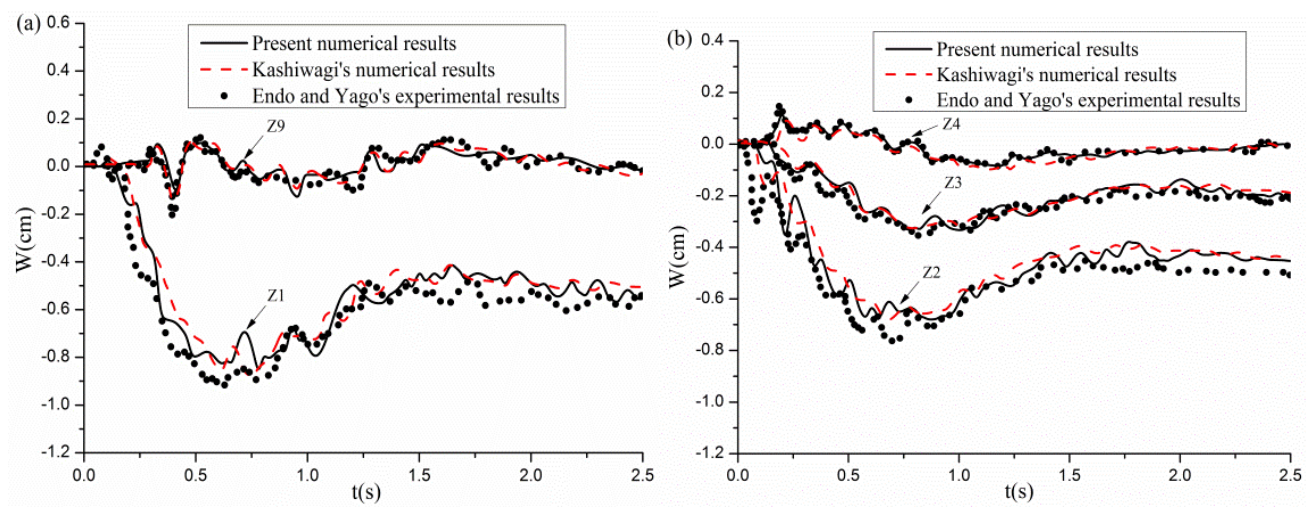

Fig. 8 Time histories of deflections at measured points during the weight drop

The deformed profiles of the VLFS during the mass drop are shown in Fig. 9. It is seen that the structural wave is transmitted at the longitudinal centerline of the plate, and the shape of the deformation is close to current static equilibrium configuration at $t=1.85 \mathrm{~s}$. The magnitude of the vertical displacements is less than $1.0 \mathrm{~cm}$. The vertical displacements of the plate is very small at $\mathrm{x}$ coordinate value along the length less than 0 , and the transient phenomena at the right edge of the VLFS is obviously seen from $t=0.21 \mathrm{~s}$ to $0.80 \mathrm{~s}$.

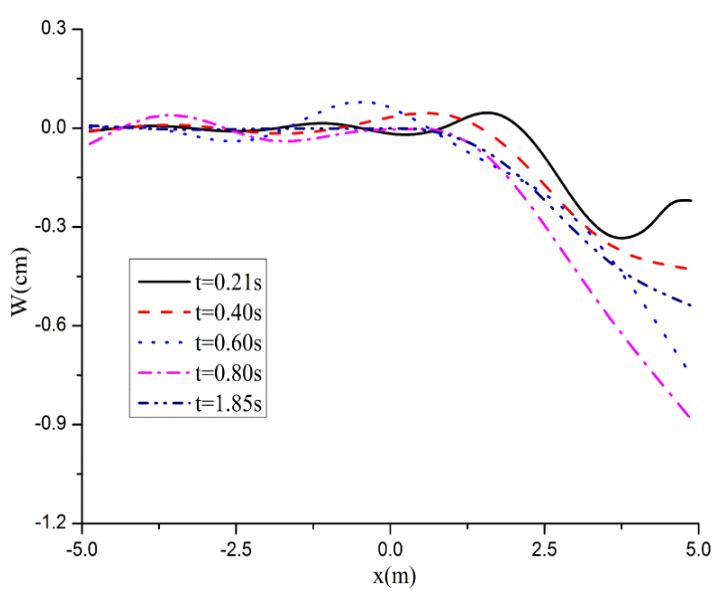

Fig. 9 Spatial profiles of the VLFS due to the mass drop 

loads in wave condition

\subsection{Landing in the still water}

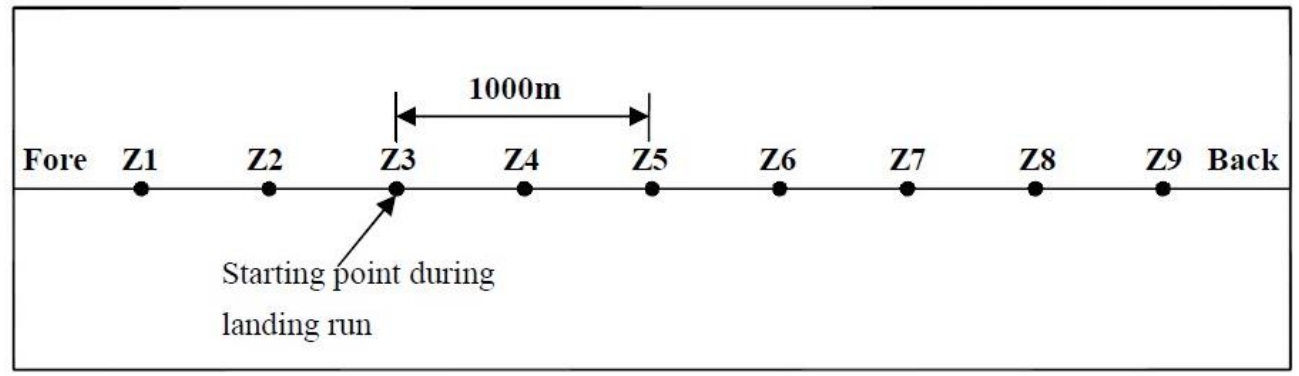

Fig. 10 The plan view of VLFS during landing run

We consider the realistic landing of an airplane on a VLFS with rectangular geometry in plane shown in Fig. 10. Here, the time-varying load is assumed to move with a constant initial acceleration $\alpha_{0}$, and the position $\xi(t)$ of the airplane and its velocity $V_{0}$ are given by

$$
\xi(t)=\xi_{0}+V_{0} t+\frac{1}{2} \alpha_{0} t^{2}
$$

$$
V(t)=V_{0}+\alpha_{0} t
$$

where $\xi_{0}$ and $V_{0}$ are the initial position and velocity, respectively. For simplicity, the load distribution is assumed to be axisymmetric about the center of the moving load $(\xi(t), 0)$. In the terms of the relationship between the moving Cartesian coordinate system $\bar{o}-\bar{x} y z$ and the polar coordinate system $\bar{o}-\bar{r} \theta z$, the external pressure distribution $P_{E}$ can be expressed as

$$
P_{E}(x, y, t)=F_{0}(t) \cdot f(\bar{r})=F_{0}(t) \cdot \frac{1}{R^{2}} e^{-\pi(\bar{r} / R)^{2}}
$$

where $\bar{r}=\sqrt{\bar{x}^{2}+y^{2}}, \bar{x}=x-\xi(t)$, and $R$ denotes the effective radius of the loading.

The total force $F_{0}(t)$ exerted by the landing or takeoff on the VLFS, can be given by the difference between the weight $W$ of the airplane and the lift force $F_{L}(t)$ :

$$
F_{0}(t)=W-F_{L}(t)
$$

$$
F_{L}(t)=\frac{1}{2} \rho_{a} V^{2}(t) A_{W} a_{L} e^{b_{L} t}
$$

where the parameters $a_{L}$ and $b_{L}$ are given as constants, $\rho_{a}$ is the density of air, and $A_{W}$ is the effective wing area of the airplane.

Table 2 Main parameters of landing run

\begin{tabular}{|l|l|l|l|}
\hline Floating airport & Airplane \\
\hline Length $(\mathrm{m})$ & 5000 & Weight $(\mathrm{KN})$ & 3867.08 \\
\hline Width $(\mathrm{m})$ & 1000 & Effective wing area $\left(\mathrm{m}^{2}\right)$ & 511.0 \\
\hline Draft $(\mathrm{m})$ & 5.0 & Effective radius $(\mathrm{m})$ & 10.0 \\
\hline Bending rigidity $(\mathrm{Nm})$ & $1.764 \times 10^{11}$ & Initial position $(\mathrm{m})$ & -1000 \\
\hline & & Initial speed $(\mathrm{m} / \mathrm{s})$ & 69.35 \\
\hline & & Acceleration $\left(\mathrm{m} / \mathrm{s}^{2}\right):$ & -1.263 \\
\hline & & $a_{L}$ in landing & 2.61 \\
\hline & & $b_{L}$ in landing & -0.212 \\
\hline
\end{tabular}


The numerical data for simulation in this paper are prepared as listed in Table 2 by referring to Kashiwagi [9]. The airplane lands at touch-down point Z3 indicated in Fig. 10 and it completes the landing run in 54.9s. The time histories of the vertical displacements at measurement point Z1, Z4 ,Z5, Z6, Z7 and Z9 obtained from the present method(direct time domain method), and the indirect time domain method used by Kashiwagi [9], are comparatively shown in Fig. 11. The simulated results generally follow the trend of the numerical curve solved by Kashiwagi [9]. It is seen that the magnitude of deflections is less than about $1.2 \mathrm{~cm}$ and is very small as compared with the length value of the runway though the airplane weight reaches about $3900 \mathrm{KN}$. Then the time histories of the vertical displacement is much smoother than the results shown in Fig. 8 during weight drop, so no higher-order deflections are found in time histories of the deflection during the landing on the platform of the VLFS. It is also interesting that the vertical displacement of measured points $\mathrm{Z} 7$ and Z9 is not so large at $t=55 \mathrm{~s}$ to $60 \mathrm{~s}$ but increases again after $t=60 \mathrm{~s}$. This is mainly due to be the radiation of structure waves which impinge the stopped airplane.
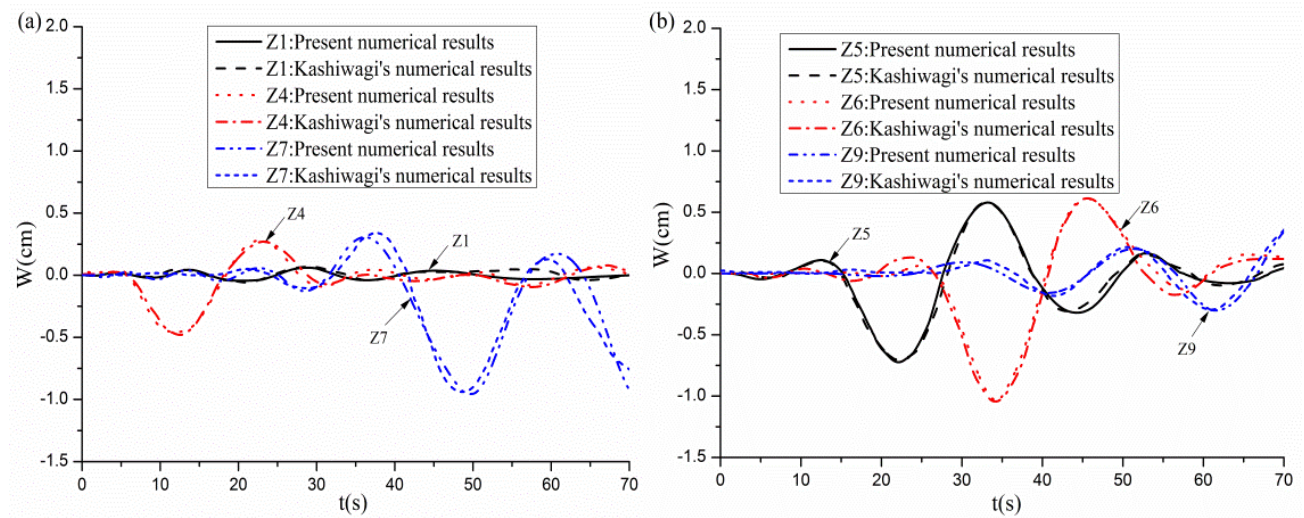

Fig. 11 Time histories of the vertical displacement subjected to landing of airplane
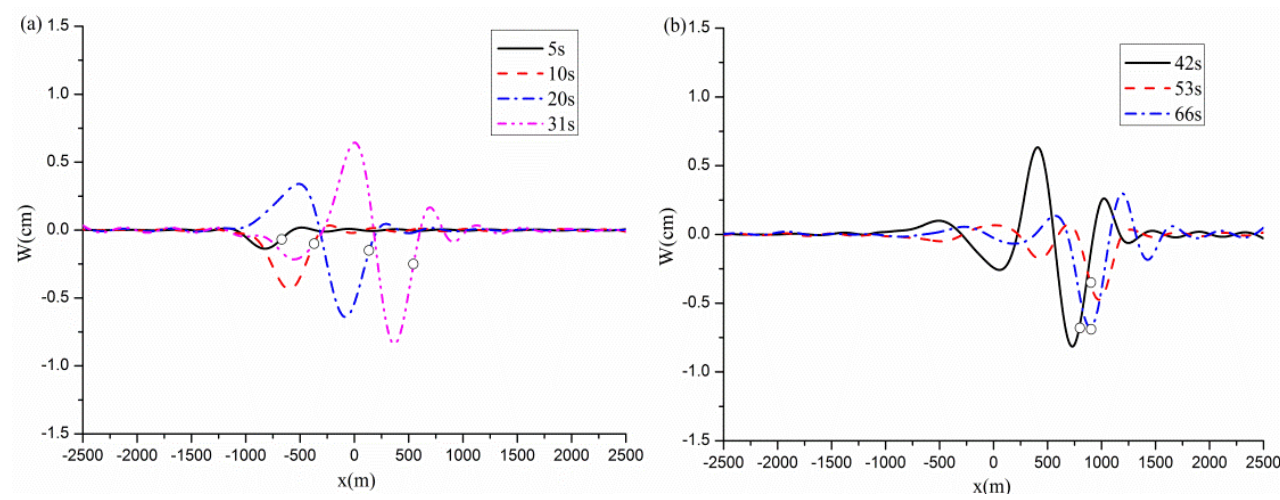

Fig. 12 Snapshots of the deflection subjected to landing of airplane

Fig. 12 shows the snapshots of the deflection along the longitudinal centerline of the runway at different times, and the corresponding positions of the airplane are expressed by circles. It is found that the structural waves run behind the airplane at time less than $42 \mathrm{~s}$, and then the waves catch up to the airplane at about time $53 \mathrm{~s}$ because of the decrease of the airplane speed. After overtaking structural waves meet the stopped airplane, partial waves are diffracted and remainder is transmitted. Thus, it is interesting to found that the deformed profiles of the runway at time $t=66 \mathrm{~s}$ is larger than that of the time $\mathrm{t}=53 \mathrm{~s}$ (see also Fig. 11). Note that the airplane seems to stay always sunken deflections of the runway during the landing run. 
loads in wave condition

\subsection{Drop test in regular wave}

In the simulation of a weight drop test in regular wave, the load force at the right hand side of Eq. (24) is divided into two stages. The regular comes first from the front side of the VLFS then the weight drops later by three cycles of the wave period. The wave length $1.0 \mathrm{~m}$, wave period is 0.8 seconds, wave height is $1 \mathrm{~cm}$ and incident angle is 0 degree.

The deformed profiles of the VLFS during the weight drop are shown in Fig. 13, where Figure 13a shows the deflections in the regular wave condition without the mass impact for $\mathrm{t}=0 \mathrm{~s}$, and the deflections in the early stage after impact for $\mathrm{t}=0.21$ and $0.41 \mathrm{~s}$. The figures tell us that the absolute values of vertical displacement at the fore-end of the VLFS in regular wave are about 10 times the ones only generated by the mass drop, however, the magnitude at the back-end is almost equivalent to the one induced by the mass drop. This means the mass impact load should be not overlooked as compared with the wave load. And the structural waves of the VLFS in wave condition are changed when the huge mass falls off on the platform of the VLFS.
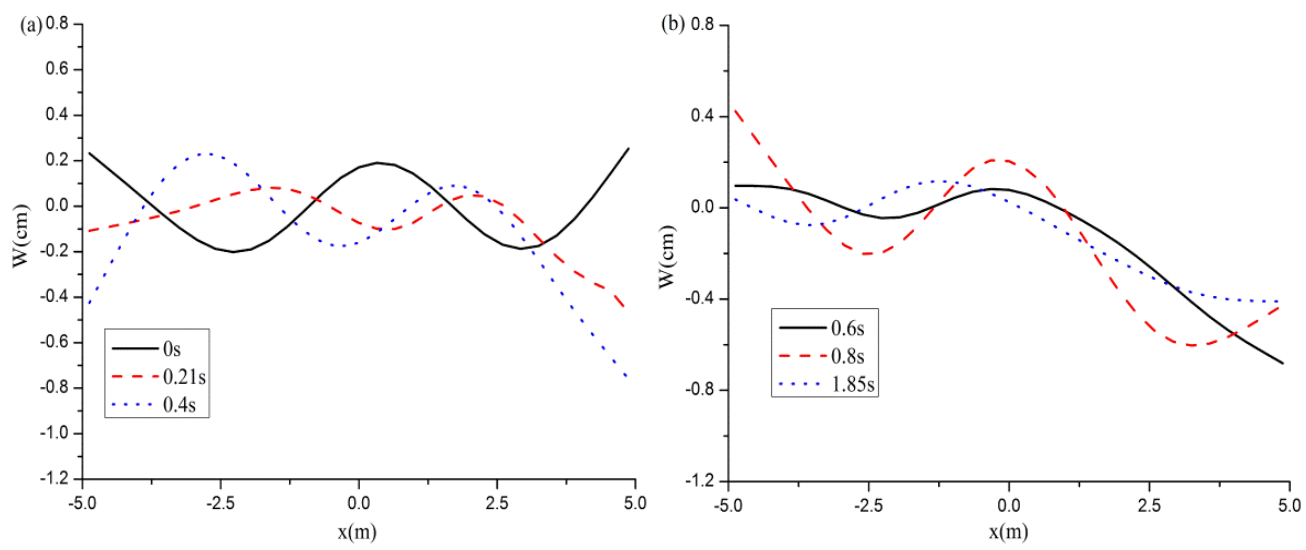

Fig. 13 Spatial profiles of the VLFS during the mass drop in regular wave conditions

\subsection{Landing in the following sea condition}

As mentioned section 5.5, the regular wave comes first from the fore-end of the VLFS, after three cycles of the wave period, the airplane touch down the runway in the following incident direction. The wavelength is $650 \mathrm{~m}$ ( 0.13 times of the runway length), period $23.6 \mathrm{~s}$, height $2.0 \mathrm{~m}$ and incident angle 0 degree.

The spatial profiles of the runway during the running are shown together with the positions of the airplane in Fig.14. From which it can be seen that the maximum vertical displacement in regular wave is $150 \mathrm{~cm}$ and is about 150 times the one induced in the still water condition. This means the wave load is dominant with compared with the landing load in the hydroelastic analysis of VLFS.

Looking the history of the vertical displacement of locations in Figure 15a and the corresponding path during landing in Figure $15 \mathrm{~b}$, it can be seen that the propagating velocity of the structural wave generated by incident wave, is slower than the landing speed of airplane in the early stage (at least up to 20s), however, when the airplane slows down, the deflections of the runway change suddenly in their magnitude and length (20s-40s shown in Fig. 15a). At the final stage of landing, speed of the airplane decreases to zero and gets left behind by structural waves. During the landing of airplane, the airplane meets two structural waves within 54.9s. And thus the vertical motion of the airplane depends mainly on the relative velocity between the structural waves and the airplane. 
A direct time-domain simulation of hydroelastic behavior of a pontoon-VLFS during unsteady external loads in wave condition
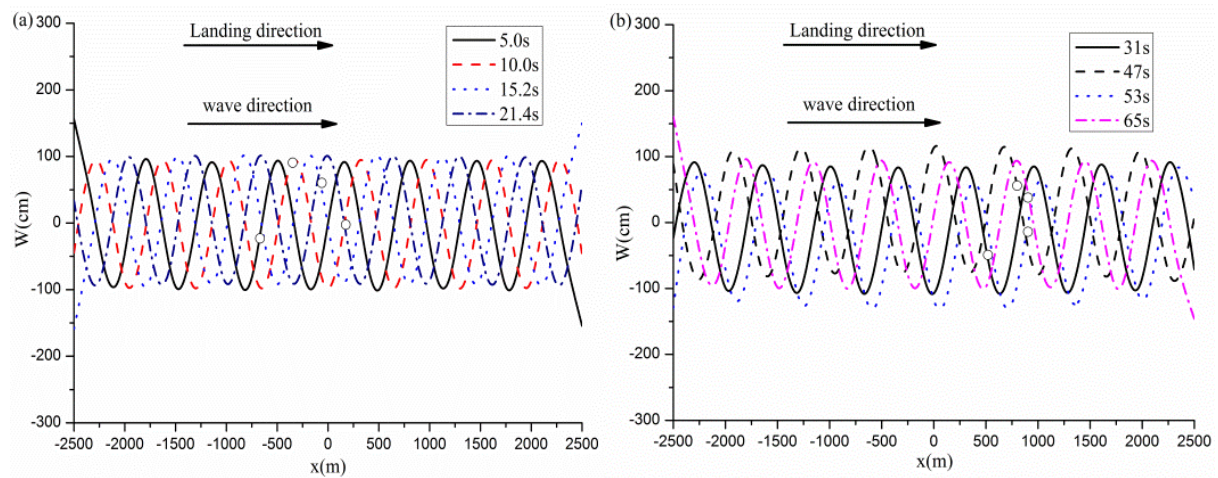

Fig.14 Spatial profiles of the deflection subjected to landing of airplane in regular wave conditions
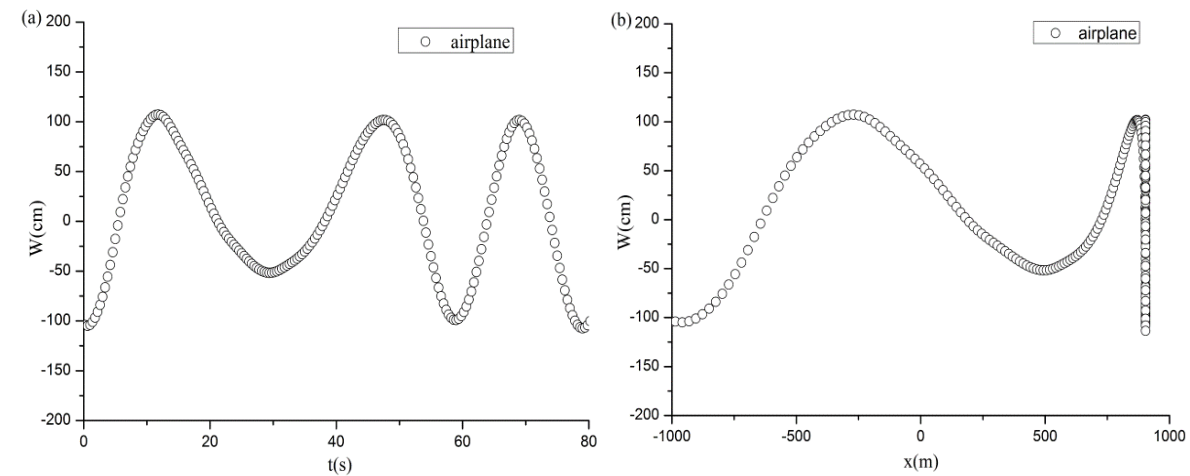

Fig. 15 (a) The time history of vertical locations of airplane in the following sea condition (b) The path of the landing run in the following sea condition

\subsection{Landing in the heading toward sea condition}

Finally, we consider the run direction of the airplane heading toward the incident wave direction for the purpose. The regular wave comes first from the back-end of the VLFS, after three cycles of the wave period, the airplane touch down the runway heading toward incident direction. The detail of VLFS and wave conditions are given in the previous section.

Fig. 16a shows the history of the vertical location during landing and Fig. 16b shows the deformed profiles of the runway in early stage and later stage of landing together with the path of the airplane. Compared with the curves in Fig. 15, it can be seen that the magnitude of the deflections is nearly the same and it has little to do with the run direction of the airplane owing to the dominant role of wave action. In this particular case, the airplane meets five structural waves within $54.9 \mathrm{~s}$ of landing run and its vertical motion mainly depends on the structural wave propagation which is raised by incident waves. In Fig. 16a, the periods of the history of vertical displacement during landing are smaller than the ones after the airplane stops run which corresponds to the speed of landing decreasing to zero.
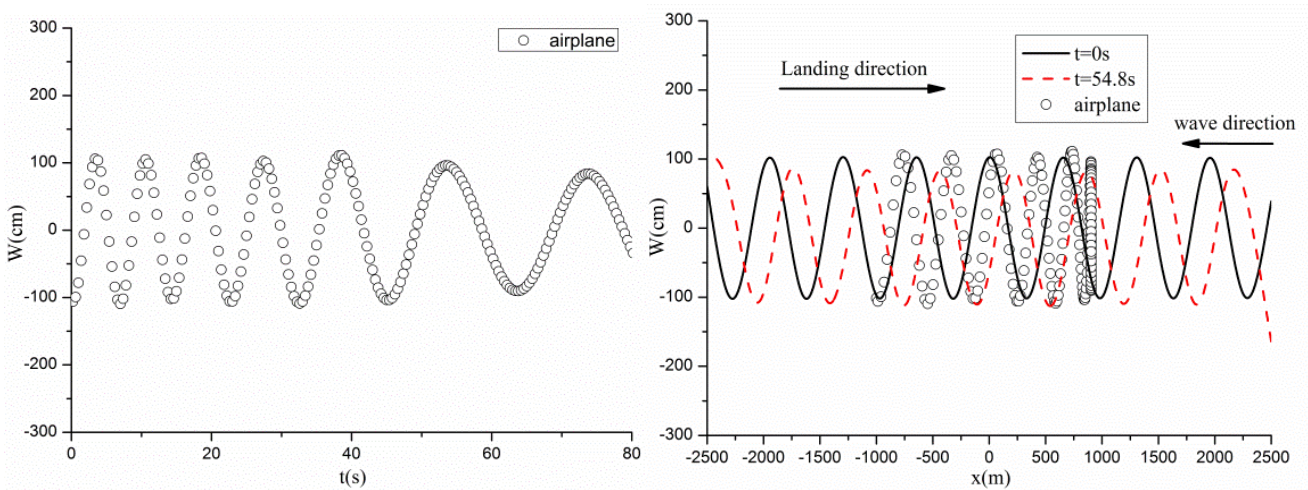

Fig. 16 (a) The time history of the vertical location during landing in the heading toward sea condition (b) The path of the Landing run in the heading toward sea condition 
A direct time-domain simulation of hydroelastic behavior of a pontoon-VLFS during unsteady external loads in wave condition

\section{Conclusions}

We have presented a time-domain hybrid finite element-boundary element (FE-ME) scheme with a robust tool for investigating the hydroelastic responses of a VLFS subjected to external loads including a weight impact onto the structure, and landing of an aircraft taking into account of the combined action of incident wave. For fluid part, the hydrodynamic forces were directly obtained by using the developed time-domain-Kelvin-source-based BEM solutions but this calculation of Green functions and its partial derivatives requires huge CPU time and memory size of computer. In order to surmount this difficulty, the bilinear and trilinear interpolation-tabulation schemes were present and applied to the fluid-structure interaction. For structural part, the coupled plate-water equation is solved using the FEM with NC-QS Mindlin plate elements. The numerical results were examined for the load cases of regular incident wave condition and of external loads including mass impact and moving load condition, respectively.

After verification by numerical and experimental results, the developed computer program was further used to simulate the hydroelastic motion of the VLFS during a weight mass drop or landing of as airplane in wave condition. From these numerical results, it was found that the vertical motion of the VLFS in regular waves is changed when the mass fall on the VLFS, even though the weight of the mass is $196 \mathrm{~N}$. However, as a result of transient responses during landing, the magnitude of deflections of runway in wave conditions is dominant as compared with the results only generated by airplane. The deflections of the runway due to the presence of airplane after landing run, can be ignored in wave condition. The vertical motion magnitude of the airplane can change abruptly during landing run in the following sea condition due to the reduction of relative velocity between the airplane and structural waves. In the heading toward incident wave direction, the airplane runs up and down on the structural waves induced by incident waves.

\section{ACKNOWLEDGMENTS}

The authors are grateful to the NSFC's Key Project (Grant No. 51490672), the National Science Foundation of China (Grant No. 51579122, 51609109, 51709118), Natural Science Found of Jiangsu Province (BK20160556), University Natural Science Research Project of Jiangsu Province (16kjb70003), Key Lab Foundation for Advanced Manufacturing Technology of Jiangsu Province (Grant No. CJ1506), China Postdoctoral Science Foundation (2017M612669), and the Fundamental Research Funds for the Central Universities (2017BQ089) for supporting this work.

\section{REFERENCES}

[1] Watanabe E., Utsunomiya T., Wang C.M., 2004. Hydroelastic analysis of pontoon-type VLFS: a literature survey, Engineering Structures. 26, 245-256. https://doi.org/10.1016/j.engstruct.2003.10.001.

[2] R. Eatock Taylor, 2007. Hydroelastic analysis of plates and some approximations, Journal of engineering mathematics 58, 267-278. https://doi.org/10.1007/s10665-006-9121-7

[3] Wu C., Watanabe E., Utsunomiya T., 1995. An eigenfunction expansion-matching method for analyzing the wave-induced responses of an elastic floating plate, Applied Ocean Research. 17, 301-310. https://doi.org/10.1016/0141-1187(95)00023-2.

[4] Watanabe E., Utsunomiya T., Wang C.M., Le T.T.H., 2006. Benchmark hydroelastic responses of a circular VLFS under wave action, Engineering Structures. 28 (2006) 423-430. https://doi.org/10.1016/j.engstruct.2005.08.014.

[5] Pham D.C., Wang C.M., Utsunomiya T., 2008. Hydroelastic analysis of pontoon-type circular VLFS with an attached submerged plate, Applied Ocean Research .30, 287-296. https://doi.org/10.1016/j.apor.2008.12.002. 
A direct time-domain simulation of hydroelastic behavior of a pontoon-VLFS during unsteady external loads in wave condition

[6] Karmakar D., Soares C.G., 2012. Scattering of gravity waves by a moored finite floating elastic plate, Applied Ocean Research. 34, 135-149. https://doi.org/10.1016/j.apor.2011.09.002.

[7] Zhao C.B., Liang R.F., Wang H.L., 2008. Influence of the external loading conditions on the hydroelastic response of floating elastic plates, Applied Ocean Research. 30, 62-71. https://doi.org/10.1016/j.apor.2008.06.001.

[8] Kashiwagi M., 2000. A time-domain mode-expansion method for calculating transient elastic responses of a pontoon-type VLFS, Journal of Marine Science and Technology. 5, 89-100. https://doi.org/10.1007/PL00010631.

[9] Kashiwagi M., 2004. Transient response of a VLFS during landing and take-off of an airplane, Journal of Marine Science and Technology.9, 14-23. https://doi.org/10.1007/s00773-003-0168-0.

[10] Cheng Y., Ji C.Y., Zhai G.J., Oleg G., 2017. Fully nonlinear numerical investigation on hydroelastic responses of floating elastic plate over variable depth sea-bottom, Marine Structures, 55, 37-61. https://doi.org/10.1016/j.marstruc.2017.04.005.

[11] Cheng Y., Zhai G.J, Ou J.P., 2014. Time-domain numerical and experimental analysis of hydroelastic response of a very large floating structure edged with a pair of submerged horizontal plates, Marine Structures, 39, 198-224. https://doi.org/10.1016/j.marstruc.2014.07.007.

[12] Watanabe E., Utsunomiya T., Tanigaki S., 1998. A transient response analysis of a very large floating structure by finite element method, Structural Eng./Earthquake Eng., JSCE. 15(2) (1998) 155-163.

[13] Qiu L.C., Liu H., 2004. Transient hydroelastic response of VLFS by FEM with impedance boundary conditions in time domain, China Ocean Engineering. 19(1), 1-9.

[14] Shin H., Lee H.Y, Lim C.G., Shin H.S., Park I.K., 2001. Hydroelastic response simulation of a VLFS in irregular waves considering taking-off and landing impact, Proceedings of OMAE'01 20th international conference on offshore mechanics and arctic engineering, Rio de Janeiro, Brazol. June. p.1-6.

[15] Malenica Š., Senjanović, I., Vladimir, N., 2013. Hydro structural issues in the design of ultra large container ships, Brodogradnja. 64(3), 323-347. https://doi.org/10.1080/17445302.2012.762728.

[16] Senjanović, I., Vladimir, N., Tomić, M., Hadžić, N., Malenica, Š, 2014. Global hydroelastic analysis of ultra large container ships by improved beam structural model, International Journal of Naval Architecture and Ocean Engineering. 6(4), 1041-1063. https://doi.org/10.2478/IJNAOE-2013-0230.

[17] Senjanović, I., Vladimir, N., Tomić, M., Hadžić, N., Malenica, Š, 2014. Some aspects of structural modelling and restoring stiffness in hydroelastic analysis of large container ships, Ships and Offshore Structure. 9(2), 199-217.

[18] Senjanović, I., Tomić, M., Vladimir, N, 2015. An advanced procedure for hydroelastic analysis of very large floating airport exposed to airplane load, Proceedings of the 7th International Conference on Hydroelasticity in Marine Technology, Split, Croatia, pp. 199-217.

[19] Utsunomiya T., Watanabe E., Wu C., Hayashi N., Nakai K., Sekita K., 1995. Wave response analysis of a flexible floating structure by BE-FE combination method, Proc. Fifth Int. Offshore and Polar Engineering Conf. p.400-5.

[20] Tay Z.Y., Wang C.D., Wang C.M., 2007. Hydroelastic response of a box-like floating fuel storage module modeled using non-conforming quadratic-serendipity Mindlin plate element, Engineering Structures. 29, 3503-3514. https://doi.org/10.1016/j.engstruct.2007.08.015.

[21] Cheng Y., Ji C.Y., Zhai G.J., Oleg G., 2016. Dual inclined perforated anti-motion plates for mitigating hydroelastic response of a VLFS under wave action, Ocean Engineering, 121, 572-591. https://doi.org/10.1016/j.oceaneng.2016.05.044.

[22] Lee D.H., Choi H.S., 2003. Transient hydroelastic response of very large floating structures by FE-BE hybrid method, In: Proc 13th Int Offshore and Polar Engineering Conf. p. 100-105.

[23] Endo H., 2000. The behavior of a VLFS and an airplane during takeoff/landing run in wave condition, Marine Structures. 13, 477-491. https://doi.org/10.1016/S0951-8339(00)00020-4.

[24] Endo H., Yago K., 1999. Time history response of a large floating structure subjected to dynamic load, J Soc Nav Archit Jpn. 186, 369-376. https://doi.org/10.2534/jjasnaoe1968.1999.186 369.

[25] Cheng Y., Ji C. Y., Zhai G. J., Oleg G., 2016. Hydroelastic analysis of oblique irregular waves with a pontoon-type VLFS edged with dual inclined perforated plates, Marine Structures, 49, 31-57. https://doi.org/10.1016/j.marstruc.2016.05.008. 
A direct time-domain simulation of hydroelastic behavior of a pontoon-VLFS during unsteady external loads in wave condition

[26] Utsunomiya T., Watanabe E., Nishimura N., 2001. Fast multipole algorithm for wave diffraction/radiation problems and its application to VLFS in variable water depth and topography, Proceedings of OMAE'01 20th international conference on offshore mechanics and arctic engineering, Rio de Janeiro, Brazol. June, p. $1-7$.

[27] Kagemoto H., Fujino M., Murai M., 1998. Theoretical and experimental predictions of the hydroelastic response of a very large floating structure in waves, Applied Ocean Research. 20 (1998) 135-144. https://doi.org/10.1016/S0141-1187(98)00017-0.

[28] Dai Y.Z., 2003. Rapid 3-dimensional hydroelastic analysis of large offshore structures, Ph.D. Dissertation, Harbin Engineering Univeisity, Harbin, china.

[29] Huang D.B., 1992. Approximation of time-domain free surface function and spatial derivatives, Shipbulding of china. 4, 16-25.

[30] Chau F.P., 1989. The second order velocity potential for diffraction of waves by fixed offshore structure, Report OEG/89/1, University College London.

[31] Wu Y.S., Xia J.Z.,. Du S.X, 1991. Two engineering approaches to hydroelastic analysis of slender ships, Proc. Of the International Union of Theoretical and Applied Mechanics, Memorial Symposium on the Dynamics of Marine Vehicles and Structures in Waves. p. 157-165.

[32] Mindlin R.D., 1951. Influence of rotator inertia and shear on flexural motions of isotropic, elastic plates, Journal of Applied Mechanics. (18), 31-8.

[33] Wehausen J.V., Laitone E.V., 1960. Surface waves, Handbuch der Physik, IX.

[34] Newman J.N., 1990. The approximation of free-surface Green functions, In: Wave Asymptotic. Manuscript for the Fritz Ursell Retirement Meeting. Cambridge: Cambridge University Press. p. 107-135 (1990).

[35] Cheng Y., Ji C.Y., Ma Z., Zhai G.J., Oleg D., 2017. Numerical and experimental investigation of nonlinear focused waves-current interaction with a submerged plate, Ocean Engineering, 135, 11-27. https://doi.org/10.1016/j.oceaneng.2017.02.038.

[36] Senjanović, I., Viadimir, N, Tomi ć, M., 2016. On new first-order shear deformation plate theories, Mechanics Research Communications. 73, 31-38. https://doi.org/10.1016/j.mechrescom.2016.02.005.

[37] Senjanobić, I., Viadimir, N, Cho, D.S., 2015. A new finite element formulation for vibration analysis of thick plates, International Journal of Naval Architecture and Ocean Engineering. ttps://doi.org/10.1515/ijnaoe-2015-0023.

[38] Senjanović, I., Viadimir, N, Cho, D.S., Jokić, M., Choi, T.M. 2015. Comparative analysis of new shear locking-free finite element with other commonly used approaches in vibration analysis of Mindlin plate, Proceedings of the 1st Pan-American Congress on Computational Mechanics-PANACM 2015, XI Argentine Congress on Computational Mechanics-MECOM, Buenos Aires, Argentina, pp. 375-388.

[39] Huang L.L., Riggs H.R. The hydrostatic stiffness of flexible floating structures for linear hydroelasticity, Marine Structures. 13, 91-106. https://doi.org/10.1016/S0951-8339(00)00007-1.

[40] Malenica S., Molin B., Tuitman J.T., Bigot F., Senjanovic I., 2009. Some aspects of hydrostatic restoring for elastic bodies, 24th IWWWFB, Saint Petersburg, Russia.

Submitted: 04.06.2017

Accepted: $\quad 23.08 .2017$.
Yong Cheng, deep_1@dlut.edu.cn, PhD

School of naval architecture and ocean engineering, Jiangsu University of Science and Technology, Zhenjiang, 212003, China

Chunyan Ji, Jichunyanjkd@163.com, Professor

School of naval architecture and ocean engineering, Jiangsu University of Science and Technology, Zhenjiang, 212003, China

Gangjun Zhai, zhai@dlut.edu.cn, Assistant Professor

Deepwater engineering research center, Dalian University of Technology,

No.2 Linggong Road, Ganjingzi District, Dalian, 116024, China

Corresponding author: Tianhui Fan, Assistant Professor, fanth@scut.edu.cn

School of civil engineering and transportation, South China University of

Technology, Guangzhou, China 